Vol. 9, $n^{\circ} 2 \mid 2005$

Varia

\title{
Andrew Maunder and Grace Moore, eds., Victorian Crime, Madness and Sensation
}

Aldershot, Ashgate, 2004

Delphine Cingal

\section{(2) OpenEdition}

\section{Journals}

Édition électronique

URL : https://journals.openedition.org/chs/308

DOI : $10.4000 /$ chs. 308

ISSN : $1663-4837$

\section{Éditeur}

Librairie Droz

Édition imprimée

Date de publication : 1 décembre 2005

Pagination : 131-133

ISBN : 978-2-600-01054-2

ISSN : 1422-0857

\section{Référence électronique}

Delphine Cingal, « Andrew Maunder and Grace Moore, eds., Victorian Crime, Madness and Sensation », Crime, Histoire \& Sociétés / Crime, History \& Societies [En ligne], Vol. 9, n² | 2005, mis en ligne le 10 février 2009, consulté le 22 mars 2022. URL : http://journals.openedition.org/chs/308 ; DOI : https:// doi.org/10.4000/chs.308

Ce document a été généré automatiquement le 22 mars 2022.

(C) Droz 


\section{Andrew Maunder and Grace Moore, eds., Victorian Crime, Madness and Sensation}

Aldershot, Ashgate, 2004

Delphine Cingal

\section{RÉFÉRENCE}

Andrew Maunder and Grace Moore, eds., Victorian Crime, Madness and Sensation, Aldershot, Ashgate, 2004.

1 Ce recueil d'essais prend le parti de traiter du crime et de la manière dont il était perçu en Angleterre à l'époque victorienne à travers sa production littéraire. En effet, les œuvres de fiction se font l'écho des fantasmes et des craintes d'une population de plus en plus urbanisée, craignant la montée de classes populaires considérées comme dangereuses.

2 La Grande-Bretagne se dota d'une force de police digne de ce nom en 1829 et d'une police judiciaire en 1842. Avec la diffusion croissante des journaux (ceux-ci proposant souvent des récits policiers ou fantastiques à leurs lecteurs), le public se tourna de plus en plus goulûment vers les crimes sensationnels, réels ou inventés. Les 'penny bloods', les 'penny dreadfuls', les 'Newgate novels', les romans policiers: toute une littérature sanglante se mit à envahir la presse.

3 Ces lectures furent parfois accusées d'être responsables de la montée - réelle ou imaginaire - de la criminalité. C'est ce va-et-vient entre la réalité et sa perception qui fait l'objet des études contenues dans ce volume.

4 La littérature policière s'inspire des progrès de la science mise au service de la justice. L'utilisation des empreintes digitales, en particulier, fascinait le public. Née de la méfiance envers les Indiens de l'administration coloniale en général, et de William Herschel en particulier, la dactyloscopie fit florès en Grande-Bretagne. Dans 'Time's 
Hand: Fingerprints, Empire and Victorian Narratives of Crime', Gita Panjabi Trelease montre comment cette technique est née et comment elle fut reprise dans les œuvres de fiction.

5 Le public voyait souvent la ville comme creuset de la criminalité. Dans son article, Dafydd Moore analyse ainsi The City of Dreadful Night, poème de James Thomson. L'aliénation, le déracinement, la misère y sont perçus comme l'origine de tous les maux.

6 Parfois, le récit reprend des faits réels, les élabore, tire des conclusions. Dallas Liddle, dans 'Anatomy of a 'Nine Days' Wonder' analyse ainsi le traitement, dans la presse, de la disparition de Benjamin Speke. La chasse à l'indice était ouverte pour trouver son cadavre.

7 Toutefois, on découvrit Speke bien en vie six semaines plus tard, en Cornouailles, déguisé en berger. Les affaires plus tragiques ne manquent pas. Ainsi, Christopher E. Forth étudie l'affaire Dreyfus à travers la presse française ('The Novelization of the Dreyfus Affair') et Nicholas Freeman se penche sur la façon dont le public percevait le tueur George Chapman qui empoisonna trois de ses épouses ('Ballad of a Demon Barber : The Criminal Career of George Chapman'.) La presse insista lourdement sur le fait que Chapman était en fait Polonais. Le côté slave de sa personnalité aurait tout pu expliquer.

8 Avant tout, c'est donc l'élément exogène à la société qui semble menacer celle-ci. Classes populaires ou étrangers venus des quatre coins de l'Empire, ce sont eux qui apparaissent comme le meurtrier potentiel. Màire nì Fhlathùin étudie, dans 'The Making of a Master Criminal: The 'Chief of Thugs' in Victorian Writings on Crime', comment l'Indien, le colonisé, devient, dans la fiction de l'époque, le criminel-type, une menace latente. Partant du personnage de Feringhea dans Le Tour du monde en quatrevingt jours, cette étude analyse le véritable personnage de Feringhea à travers les rapports de W.H. Sleeman. C'est cette même méfiance de l'étranger qui perce dans Blind Love de Wilkie Collins. L'Irlandais devient le symbole d'une race dangereuse (voir 'Furious Passions of the Celtic Race: Ireland, Madness and Wilkie Collins's Blind Love' de Maria K. Bachman.) À l'opposé, le criminel peut être n'importe qui dans la foule. Karen Odden se penche ainsi sur la criminalité en col blanc dans The Way We Live Now d'Anthony Trollope.

9 L'image de la femme dégénérée, renonçant à son rôle de vierge ou de mère, hante la littérature victorienne. Le personnage de Constance Kent, célèbre meurtrière de son petit frère, a influencé de nombreux auteurs. Jane Sturrock, dans 'Murder, Genre, and Popular Fiction by Women in the 1860s: Braddon, Oliphant, Yonge', montre comment trois récits policiers ont été directement inspirés de l'affaire de Constance Kent : Lady Audley's Secret de Mary Elizabeth Braddon, Salem Chapel de Margaret Oliphant et The Trial de Charlotte Yonge. La transgression féminine menace l'équilibre de la société. En particulier ses appétits sexuels débordants. Dans East Lynne, Ellen Wood décrit la chute d'une aristocrate dont la liaison adultérine aboutit au divorce. (Voir l'article d'Andrew Maunder, 'Stepchildren of Nature: East Lynne and the Spectre of Female Degeneracy'). Le viol d'enfants et la prostitution de mineurs fait l'objet d'une apparition métaphorique dans Dracula, lorsque Lucy Westenra, devenue vampire, s'en prend à de tout petits enfants (voir 'Vamping the Children: The 'Bloofer Lady', the 'London Minotaur' and Child-Victimization in Late Nineteenth Century England' de Leslie Ann Minot.) Dans 'The Inside Story: Crime, Convicts and Careers for Women', Barbara 
Onslow montre l'inquiétude de certaines journalistes pour le sort des femmes des classes populaires. Sans occupations rémunérées possibles, elles devaient souvent se prostituer. Harriet Martineau proposa alors que celles-ci, dans les prisons, puissent obtenir un travail, puis qu'elles continuent à pouvoir être employées à leur sortie.

La sexualité déviante est centrale à la littérature victorienne. Elle apparaît toutefois de manière imagée (le vampire, le monstre, etc.). Grace Moore propose ainsi une explication assez intéressante à la relation entre Jekyll et Hyde. Si Hyde représente la face sombre de Jekyll, c'est que ce dernier a quelque chose à cacher ('Something to Hyde' pour reprendre le jeu de mots de Grace Moore dans le titre de son article). Analysant les travaux de pseudo-scientifiques sur les conséquences physiques et morales de l'onanisme, Grace Moore montre Hyde comme la face cachée du masturbateur.

11 Ce recueil propose également plusieurs études sur des criminalités plus marginales, voire folkloriques. L'article de John Plunkett, 'Regicide and Reginamania: G.W.M. Reynolds and The Mysteries of London', décrit la fascination qu'éprouvait le peuple britannique pour sa jeune reine et la folie collective qui régnait depuis son accession au trône. Par conséquent, plusieurs hommes se déclarèrent amoureux d'elle, parmi lesquels 'Boy Jones', personnage repris par G.W.M. Reynolds dans ses Mysteries of London. À travers le regard de Holford, le lecteur pénètre la vie quotidienne de la reine. Lorsque le Prince Albert découvre l'intrus et l'expulse du palais, celui-ci est saisi de haine et décide de commettre un régicide. Dans 'Black Markets and Cadaverous Pies: The Corpse, Urban Trade and Industrial Consumption in the Penny Blood', Sally Powell analyse le phénomène des "résurrectionnistes" (des voleurs de cadavres qui fournissaient les scientifiques et les médecins pour les autopsies) et leur perception dans la série Life in London de Henry Thornley. Le cadavre devient alors une marchandise comme une autre, objet de commerce, tarifé en fonction de son état.

Ce recueil propose ainsi une vision variée et passionnante des divers aspects de la criminalité au XIX ${ }^{e}$ siècle, à travers la fiction de l'époque. Il s'agit d'un travail particulièrement varié et riche.

\section{AUTEURS}

\section{DELPHINE CINGAL}

(Université Panthéon-Assas-Paris II), dcingal@club-internet.fr 